\title{
Basilar Rales
}

National Cancer Institute

\section{Source}

National Cancer Institute. Basilar Rales. NCI Thesaurus. Code C61454.

Abnormal breath sounds (crackles) heard on auscultation only in the bases of the lungs.

They indicate inflammation, fluid, or infection in the air sacs of the lung. 